\title{
DEEPLY VIRTUAL AND EXCLUSIVE PRODUCTION OF THE OMEGA MESON
}

\author{
M. GARÇON, L. MORAND, D. DORÉ, J.-M. LAGET, S. MORROW and F. SABATIÉ \\ On behalf of the CLAS Collaboration \\ DAPNIA/SPHN, CEA-Saclay, 91191 Gif-sur-Yvette, France
}

\begin{abstract}
Exclusive $\omega$ electroproduction off the proton was measured at the highest possible fourmomentum transfer with the (close to) $6 \mathrm{GeV}$ beam now available at CEBAF. Cross sections are presented, together with an analysis of the $\omega$ spin density matrix elements. Indications are that $\pi^{0}$ exchange in the $t$-channel (or rather the exchange of the corresponding saturating Regge trajectory) seems to dominate the process $\gamma^{*} p \rightarrow \omega p$, even for photon virtuality $Q^{2}$ as large as $5 \mathrm{GeV}^{2}$. Contributions of the handbag type, related to Generalized Parton Distributions in the nucleon, are therefore difficult to extract from this particular process.
\end{abstract}

Keywords: Vector meson electroproduction; omega; Spin density matrix elements.

\section{Introduction}

Most real photon induced reactions, over a wide energy range (from CEBAF to HERA energies), are well described by the exchange in the $t$-channel of a few Regge trajectories, This is especially true for the forward, diffractive, cross sections. The exchange of the Pomeron (or its realization into two gluons) dominates at high energies, while the exchange of meson Regge trajectories $\left(\pi, \sigma, f_{2}\right)$ takes over at low energies. At $\gamma p$ energies of a few $\mathrm{GeV}, \omega$ photoproduction off a proton is dominated by $\pi^{0}$ exchange in the $t$-channel. The use of saturating Regge trajectories is very successfull ${ }^{1}$ in describing recent photoproduction data ${ }^{2}$ at large momentum transfer $t$. This is a simple and economic way to parameterize hard scattering mechanisms. Extending these measurements to the virtual photon sector opens up the way to tune the hadronic component of the exchanged photon, to see to what extent $\pi^{0}$ exchange survives, and to observe hard scattering mechanisms with the help of a second hard scale, the virtuality $Q^{2}$ of the photon.

The study of the $x_{B}$ and $t$ dependence of these reactions in the Bjorken regime holds promise, through perturbative QCD, to access the so-called Generalized Parton Distributions (GPD) of the nucleon. In this kinematical regime, the process under study may be represented by a handbag diagram and its amplitude factorizes into a "hard" process $\gamma^{*} q \rightarrow q m$ and a "soft" one containing the new information on the nucleon, the GPDs. Note that the factorization applies only to the 
$\gamma_{L}^{*} \rightarrow m_{L}$ (longitudinal or zero helicity) transition which is the leading one in the Bjorken regime ${ }^{3}$. The actual determination of the GPDs will require an extended and ambitious experimental program. Our present goal is to make a step in that direction, testing whether the handbag contribution describes the considered exclusive processes. The dominance of the handbag diagram is expected to be reached at a higher $Q^{2}$ for meson production than for photon production (DVCS). Nevertheless, vector meson production is an important complement to DVCS since it singles out the GPDs sensitive to the quark angular momentum through Ji's sum rule 4 and allows, in principle, for a flavor decomposition of these distributions.

\section{The Experiment}

The CEBAF experiment $99-105{ }^{5}$ was the first " $6 \mathrm{GeV}$ " experiment proposed at CLAS, with the aim to measure the exclusive electroproduction of the vector mesons $\rho, \omega, \phi$ on the nucleon in the Bjorken regime $\left(Q^{2}\right.$ large and $x_{B}$ finite). In the specific case of $\omega$ production, its main goal was to test which of the two descriptions, Regge type or handbag dominance, applies in our kinematical regime.

The $5.754 \mathrm{GeV}$ CEBAF electron beam was incident upon a $5 \mathrm{~cm}$ long liquid hydrogen target. The scattered electrons, recoil protons and charged pions were detected in the CLAS spectrometer ${ }^{6}$. Events corresponding to $\gamma^{*} p$ energies smaller than $1.8 \mathrm{GeV}$, or scattered electron energies smaller than $0.8 \mathrm{GeV}$, were rejected from the analysis.

The $\omega$ meson was identified through its decay $\omega \rightarrow \pi^{+} \pi^{-} \pi^{0}$ and missing mass techniques. The cross sections were extracted from the analysis of the $e p \rightarrow e p \pi^{+} X$ configurations (i.e. $e, p$ and $\pi^{+}$detected in CLAS), while the analysis of the $\omega$ polarization required the determination of the decay plane (in the $\omega$ rest frame) and therefore the detection of two pions. In that case, the $e p \rightarrow e p \pi^{+} \pi^{-} X$ configurations were used. In both cases, the CLAS acceptance was calculated in multidimensional elementary bins using simulation, which necessitated extensive computer calculations. Details of the data analysis are presented elsewhere ${ }^{7}$.

\section{Cross sections}

The reduced cross sections for the process $\gamma^{*} p \rightarrow \omega p$ integrated over $t\left(-2.7 \mathrm{GeV}^{2}<\right.$ $t<t_{0}\left(Q^{2}, x_{B}\right)$ ) and over $\Phi$ (the angle between the leptonic and hadronic planes) are presented in Figure 1. The errors are dominated by systematic uncertainties coming mostly from the incomplete knowledge of acceptance and from the subtraction of non-resonant three-pion background. The results extend to $Q^{2}$ values as high as 5 $\mathrm{GeV}^{2}$. For each value of $x_{B}$, the $Q^{2}$ range is limited by the beam energy, and not by the available statistics. All these cross sections are significantly higher than the contribution from longitudinal virtual photons estimated in a GPD model ${ }^{9}$.

The $t$-dependence of the cross section was illustrated and discussed in a recent publication ${ }^{10}$. Remarkably, the high- $t$ behaviour of the cross sections is nearly $Q^{2}$ independent. A Regge-based model, where the process is dominated by $\pi^{0}$ exchange, 


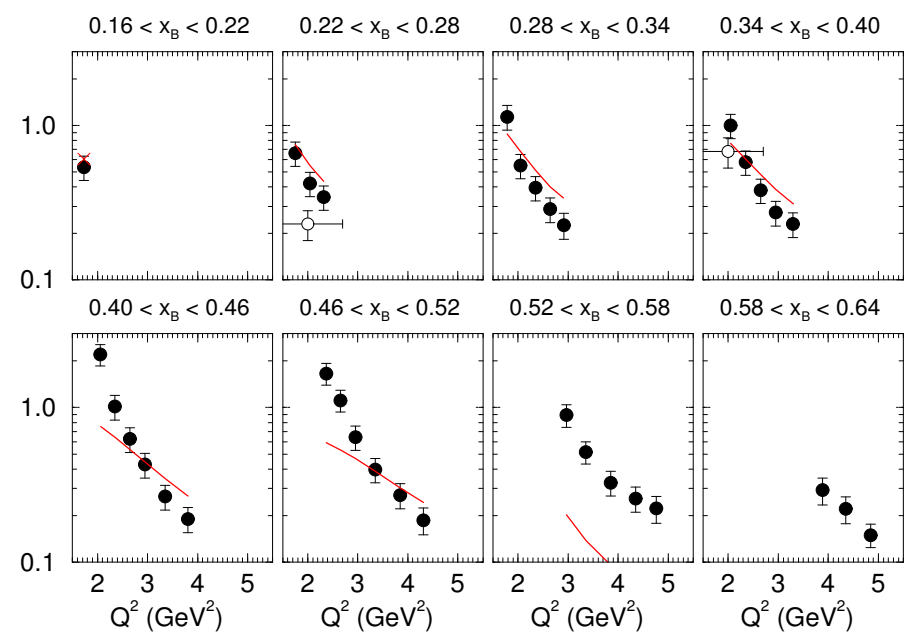

Fig. 1. Reduced total cross sections $\sigma\left(\gamma^{*} p \rightarrow \omega p\right)$, in $\mu$ barn, as a function of $Q^{2}$ for 8 different bins in $x_{B}$. The error bars include statistical and systematic uncertainties. Open circle from Cornell experiment ${ }^{8}$. Curve from model discussed in text ${ }^{10}$.

reproduces very well all data, provided a $t$ dependence is introduced in the $\gamma \pi \omega$ form factor, suggesting a coupling to a point-like object at high values of $|t|^{10}$.

\section{Spin density matrix elements}

We extracted from the $\omega$ decay distribution 15 spin density matrix elements (SDME) describing the $\omega$ polarization in the final state. Several of the SDMEs linked to helicity flip amplitudes (between the virtual photon and the outgoing $\omega$ ) were shown to be non-zero over our whole kinematical range (see Figure 2). Thus the $s$-channel helicity does not appear to be conserved, which would be the case if the handbag diagram were dominant. Furthermore, a specific combination of the SDMEs points to the exchange of unnatural parity particle in the $t$-channel, which is consistent with the dominance of the $\pi^{0}$ exchange contribution.

\section{Conclusions}

High statistics $e p \rightarrow e p \omega$ data were presented, covering a large kinematical domain. Our preliminary conclusions are then:

- The cross sections are unexpectedly high at large values of $|t|$.

- $s$-channel helicity is not conserved, which makes it impossible to separate the transverse and longitudinal cross sections using only the $\omega$ spin density matrix elements.

- The $\pi^{0}$ exchange contribution appears to be dominant over our kinematical range, and this triggered new calculations within a Regge model ${ }^{10}$. 
$5.2 \uparrow \mathrm{Q}^{2}\left(\mathrm{GeV}^{2}\right)$

4.1

3.5

2.9
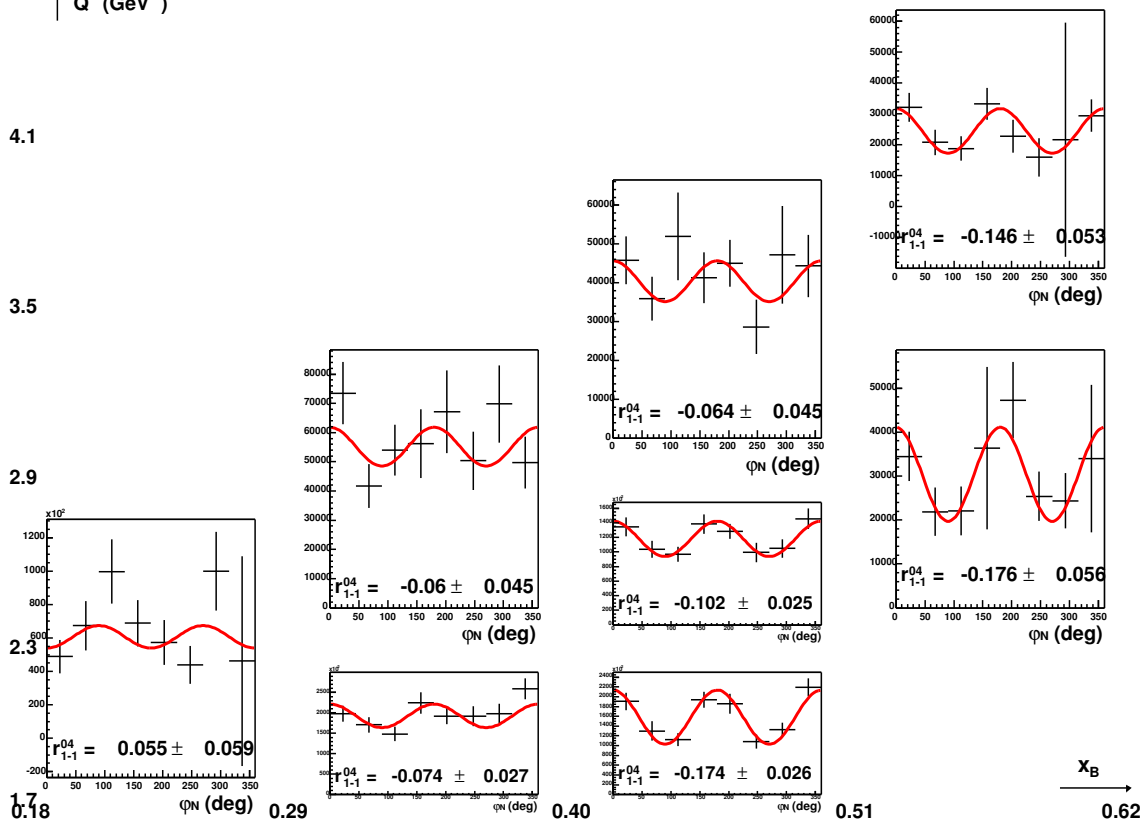

Fig. 2. Dependence of cross section (in arbitrary units) on $\varphi_{N}$, one of the angles characterizing the $\omega$ decay plane orientation. Results are presented for 8 bins in $\left(Q^{2}, x_{B}\right)$, the area for each graph corresponding to the $\Delta Q^{2} \times \Delta x_{B}$ integration range. All data are integrated in $t\left(-t<2.7 \mathrm{GeV}^{2}\right)$. The curve corresponds to a fit with the expected distribution ${ }^{11} \mathcal{W}\left(\varphi_{N}\right) \propto\left[1-2 r_{1-1}^{04} \cos 2 \varphi_{N}\right]$. The SDME $r_{1-1}^{04}$ and its statistical error are indicated on each distribution. The non-zero values point to the non-conservation of $s$-channel helicity.

- The handbag diagram may contribute to the process, but this contribution, in the specific case of $\omega$ production, may be too small compared to the $\pi^{0}$ exchange to be extracted. From this point of view, the $\rho$ electroproduction seems more promising and is being analyzed.

\section{References}

1. F. Cano and J.-M. Laget, Phys. Rev. D 65, 074022 (2002).

2. M. Battaglieri et al., Phys. Rev. Lett. 90, 022002 (2003).

3. J.C. Collins, L. Frankfurt, and M. Strikman, Phys. Rev. D 56, 2982 (1997).

4. X. Ji, Phys. Rev. Lett. 78, 610 (1997).

5. M. Guidal, M. Garçon, E. Smith and the CLAS collaboration, CEBAF experiment 99-105 (1999).

6. B.A. Mecking et al., Nucl. Instr. and Meth. A503, 513 (2003).

7. L. Morand, Thèse de Doctorat, Université Denis Diderot-Paris 7, Report DAPNIA03-09-T, 2003; L. Morand et al., in preparation.

8. D.G. Cassel et al., Phys. Rev. D 24, 2787 (1981).

9. M. Vanderhaeghen et al., Phys. Rev. D 60, 094017 (1999); and work in progress.

10. J.-M. Laget, Phys. Rev. D 70, 054023 (2004); and private communications.

11. K. Schilling and G. Wolf, Nucl. Phys. B61, 381 (1973). 